\title{
Comparison of effects of low-level laser therapy and extracorporeal shock wave therapy in calcaneal spur treatment: A prospective, randomized, clinical study
}

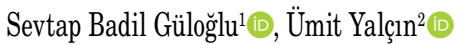 \\ ${ }^{1}$ Department of Physical Medicine and Rehabilitation, Kafkas University Faculty of Medicine, Kars, Turkey \\ ${ }^{2}$ Department of Physical Medicine and Rehabilitation, Medicana International Hospital Istanbul, Istanbul, Turkey
}

Received: September 13, 2019 Accepted: April 14, 2020 Published online: May 25, 2021

\begin{abstract}
Objectives: In this study, we aimed to evaluate and compare the efficacy of low-level laser therapy (LLLT) and extracorporeal shock wave therapy (ESWT) in the treatment of calcaneal spurs.

Patients and methods: A total of 62 patients (14 males, 48 females; mean age: $47.6 \pm 11.7$ years; range, 18 to 70 years) who were diagnosed with calcaneal spurs based on clinical examination and plain radiography between April 2019 and September 2019 were included in this study. A total of 15 sessions of plantar fascia gastroc-soleus stretching exercises and cold pack treatments were given to both groups. The LLLT (904 $\mathrm{nm}$ wavelength, $3,000 \mathrm{~Hz}, 8 \mathrm{~J} / \mathrm{cm}^{2}$ dose to the painful heel area and insertion of the plantar fascia on the medial calcaneal area, five points for a total of $5 \mathrm{~min}$ for three weeks) was applied to the first group ( $\mathrm{n}=31$ ), whereas ESWT (10 Hz, 2,000 shock waves with a 2.5 bar pressure into the areas of the painful heel, insertion of the plantar fascia on the medial calcaneal area) was applied the second group ( $\mathrm{n}=31$ ). All patients were evaluated using the Visual Analog Scale (VAS) and Foot Function Index (FFI) before and after treatment.

Results: In both groups, the median VAS and FFI scores after treatment showed a significant improvement, compared to pre-treatment scores $(\mathrm{p}=0.001)$. There was no significant difference between the groups in terms of the median post-treatment VAS scores $(\mathrm{p}>0.05)$. In the ESWT group, the median FFI pain and total scores after treatment were significantly lower than in the LLLT group ( $\mathrm{p}=0.033$ ). The change in the median FFI pain and total scores were significantly higher in the ESWT group $(\mathrm{p}=0.046)$.

Conclusion: Both treatment modalities are effective and not superior to each other in terms of disability and activity limitation reduction, although a greater improvement in the FFI pain and total scores can be achieved with the ESWT. Based on these findings, we recommend both non-invasive treatment methods to be used in the treatment of calcaneal spurs in the clinical practice.
\end{abstract}

Keywords: Calcaneal spur, extracorporeal shock wave therapy, low-level laser therapy.

Calcaneal spur (CS), which is one of the most common causes of localized pain, can cause serious discomfort and limitations in daily life. ${ }^{[1]}$ Symptoms include pain in the heel region, in particular while walking and rising from a prone position after a long period, such as getting out of bed in the morning. Calcaneal spur, which affects 15 to $20 \%$ of the general population, can be seen at any age ${ }^{[2]}$ while symptomatic $\mathrm{CS}$ is more common in overweight elderly women. ${ }^{[3]}$
Diagnosis of CS is based on medical history and clinical examination findings. Palpation of the medial tubercle of the calcaneus may cause tenderness and pain. Diagnostic imaging methods detect the presence of specific anatomical structures and plain radiographs are helpful in the diagnosis of CS. ${ }^{[4]}$

Conservative treatment modalities include resting, cold application, stretching exercises, non-steroidal

Corresponding author: Sevtap Badil Güloğlu, MD. SBÜ Antalya Eğitim ve Araştırma Hastanesi, Fizik Tedavi ve Rehabilitasyon Kliniği, 07100 Muratpaşa, Antalya, Türkiye. e-mail: drsevtapbadil@hotmail.com 
anti-inflammatory drugs, splint therapy, shoe modification, therapeutic ultrasound, extracorporeal shock wave therapy (ESWT), and low-energy laser therapy (LLLT). ${ }^{[5,6]}$ In addition, steroid injections and platelet-rich plasma injections can be performed. Conservative methods are successful in approximately $90 \%$ of patients in the treatment of $C S,{ }^{[1]}$ whereas open or endoscopic surgical techniques are required in patients who do not benefit from conservative methods. However, surgical interventions should be considered carefully due to the prolonged recovery time, impaired foot biomechanics, and the possibility of complications and recurrences. ${ }^{[7]}$ There are many studies in the literature investigating the efficacy of different treatment modalities for CS treatment; however, the most effective method of treatment is still unclear. $^{[8]}$

Several studies have shown that ESWT is effective in reducing pain related to $\mathrm{CS}^{[1,9-11]}$ The pressure waves of ESWT pass through fluids and soft tissues, exerting their effects on areas of impedance change, such as bones and soft tissue spaces. These waves have mechanical and cellular effects, and the most important of these are transient damage to the neuronal cell membrane or increased permeability. These mechanisms can facilitate cellular regeneration in neovascularization of tissues which explain the analgesic effects of ESWT. ${ }^{[10]}$

Low-level laser therapy is an increasingly used treatment modality in many musculoskeletal diseases. Recent studies have demonstrated that it is effective in the treatment of plantar fasciitis. ${ }^{[12-14]}$ It is used to accelerate tissue healing and reduce pain and inflammation. ${ }^{[15]}$ It promotes cell proliferation and tissue regeneration through a photobiostimulation effect. ${ }^{[16]}$ It has been suggested that treatment protocols and standardization of dose according to the disease directly affect the treatment success, ${ }^{[17]}$ although no studies have been carried out on CS, yet.

To the best of our knowledge, despite the increasing popularity of LLLT and ESWT, there is no randomized-controlled trial available comparing the effectiveness of these methods in the treatment of CS. In the present study, therefore, we aimed to evaluate and compare the efficacy of ESWT and LLLT in the treatment of CS.

\section{PATIENTS AND METHODS}

This prospective, randomized, clinical study was carried out Kafkas University Faculty of Medicine and Medicana International Hospital Istanbul,
Physical Medicine and Rehabilitation outpatient clinic between April 2019 and September 2019. A total of 79 patients aged between 18 and 70 years, who were diagnosed with a CS based on clinical examination and plain radiography were recruited. Nine patients were excluded and eventually 66 patients were volunteered to participate. Inclusion criteria were as follows: 18 to 70 years of age, presence of heel pain for at least four weeks, diagnosis of CS, receiving no medical treatment, injection, physical or surgical treatment (treatment-naive) for the last four weeks. Exclusion criteria were as follows: acute trauma to the foot, history of fracture or surgery, lower extremity neurological deficit, polyneuropathy, presence of lumbar pathology findings related to foot pain, inflammatory rheumatological disease, history of anticoagulant use, tumor, thrombosis, soft tissue or bone infection, acute inflammation, pregnancy, lactation, epilepsy, presence of a pacemaker, hematological disease, coagulation disorder, hemoglobin $<11 \mathrm{~g} / \mathrm{dL}$, platelet count $<150,000 / \mathrm{mm}^{3}$, and skin disorder. Prior to the study, all patients were informed about the nature of the study and a written informed consent was obtained from each patient on a voluntary basis. The study protocol was approved by the Kafkas University Faculty of Medicine's Ethics Committee (date/no: 26.12.2018/19). The study was conducted in accordance with the principles of the Declaration of Helsinki.

The patients were randomly divided into two groups using the stratified randomization according to sex and age. A total of 15 sessions of cold pack (20 $\mathrm{min})$ and plantar fascia gastroc-soleus stretching exercises were given to both groups. The first group $(n=32)$ received LLLT (ILUX Yag 1064; Mectronic Medicale srl, Bergamo, Italia) (904 $\mathrm{nm}$ wavelength, $3,000 \mathrm{~Hz}, 8 \mathrm{~J} / \mathrm{cm}^{2}$ dose to the painful heel area and insertion of the plantar fascia on the medial calcaneal area, five points for a total of $5 \mathrm{~min}$ for three weeks), ${ }^{[18]}$ while the second group $(n=35)$ received ESWT (Medical Italia Shock Med; EME srl, Bergamo, Italia) $(10 \mathrm{~Hz}, 2,000$ shock waves with a $2.5 \mathrm{bar}$ pressure into the areas of the painful heel, insertion of the plantar fascia on the medial calcaneal area, three sessions at one-week intervals). ${ }^{[13]}$

Baseline demographic and clinical characteristics of the patients were documented. All patients were evaluated using the Visual Analog Scale (VAS)-pain and Foot Function Index (FFI) before and after the treatment. Using the VAS, each patient was asked to mark the severity of pain on a $100-\mathrm{mm}$ line with "no pain" on one end and "most unbearable pain" 
on the other end. ${ }^{[19]}$ The distance from the starting point of the pain to the point marked by the patient was recorded. Higher scores indicate a greater pain. The FFI was originally developed to assess foot pain, disability and activity limitation, and its Turkish validity and reliability studies were performed by Anaforoğlu Külünkoğlu et al. ${ }^{[20]}$ It consists of 23 items including nine items for the pain, nine items for the disability, and five items for the activity restriction. Each item is scored on a 10-point scale. To calculate the total score, the scores are summed and divided by the total maximum score which can be taken from all items, and multiplied by 100 . Higher scores indicate weak foot health.

\section{Statistical analysis}

Statistical analysis was performed using the IBM SPSS version 22.0 software (IBM Corp., Armonk, NY, USA). Quantitative data were expressed in mean \pm standard deviation (SD) or median (min-max), while qualitative data were expressed in number and frequency. The normality distribution of variables was examined using the Shapiro-Wilk test. An independent sample t-test and Mann-Whitney $U$ test were used for the analysis of quantitative independent data. The Wilcoxon test was used for the analysis of dependent quantitative data and the Pearson chi-square test was carried out for the analysis of qualitative independent data. A $p$ value of $<0.05$ was considered statistically significant.

\section{RESULTS}

Initially, 66 patients who met the inclusion criteria were included in the study. However, three patients discontinued the treatment period $(n=1$ in the LLLT group and $n=2$ in the ESWT group), and one patient was lost to follow-up after the treatment $(\mathrm{n}=1$ in the ESWT group). Finally, a total of 62 patients with CS (14 males, 48 females; mean age: $47.6 \pm 11.7$ years; range, 18 to 70 years) including 31 patients in each group were analyzed. Baseline demographic and clinical characteristics of the patients are summarized in Table 1. The mean BMI was $32.4 \pm 5.2$ (range, 24.5 to 42.2$) \mathrm{kg} / \mathrm{m}^{2}$ in the LLLT group and $29.8 \pm 4.8$ (range, 22.4 to 42.9 ) $\mathrm{kg} / \mathrm{m}^{2}$ in the ESWT group ( $\mathrm{p}=0.051)$. The median symptom duration was 18 (range, 1 to 120) months in the LLLT group and 20 (range, 5 to 120) months in the ESWT group ( $\mathrm{p}=0.949)$. There was no significant difference in the baseline demographic and clinical characteristics of the patient groups.

The median (min-max) VAS, FFI pain, FFI disability, FFI activity limitation, and total FFI scores before treatment were $8.0(5.0-10.0), 75.7$ (31.1-100), 68.9 (24.0-100.0), 34.0 (4.0-88.0), 57.4 (25.7-94.5), respectively. There was no significant difference in the baseline VAS and FFI scores between the patient groups (Table 2). However, after the treatment, the median VAS and FFI scores improved significantly in both groups $(\mathrm{p}=0.001)$, although there was no

TABLE 1

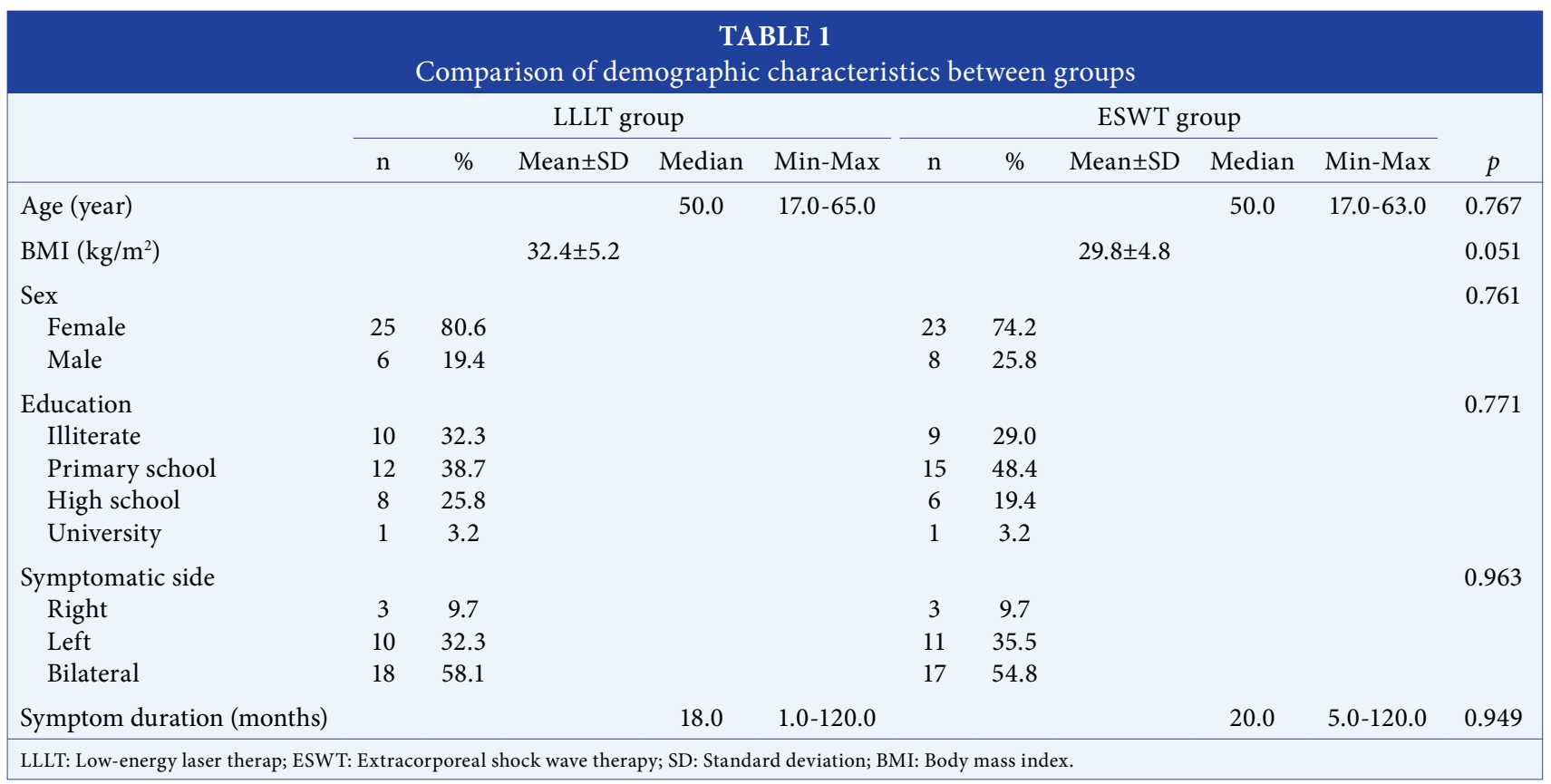




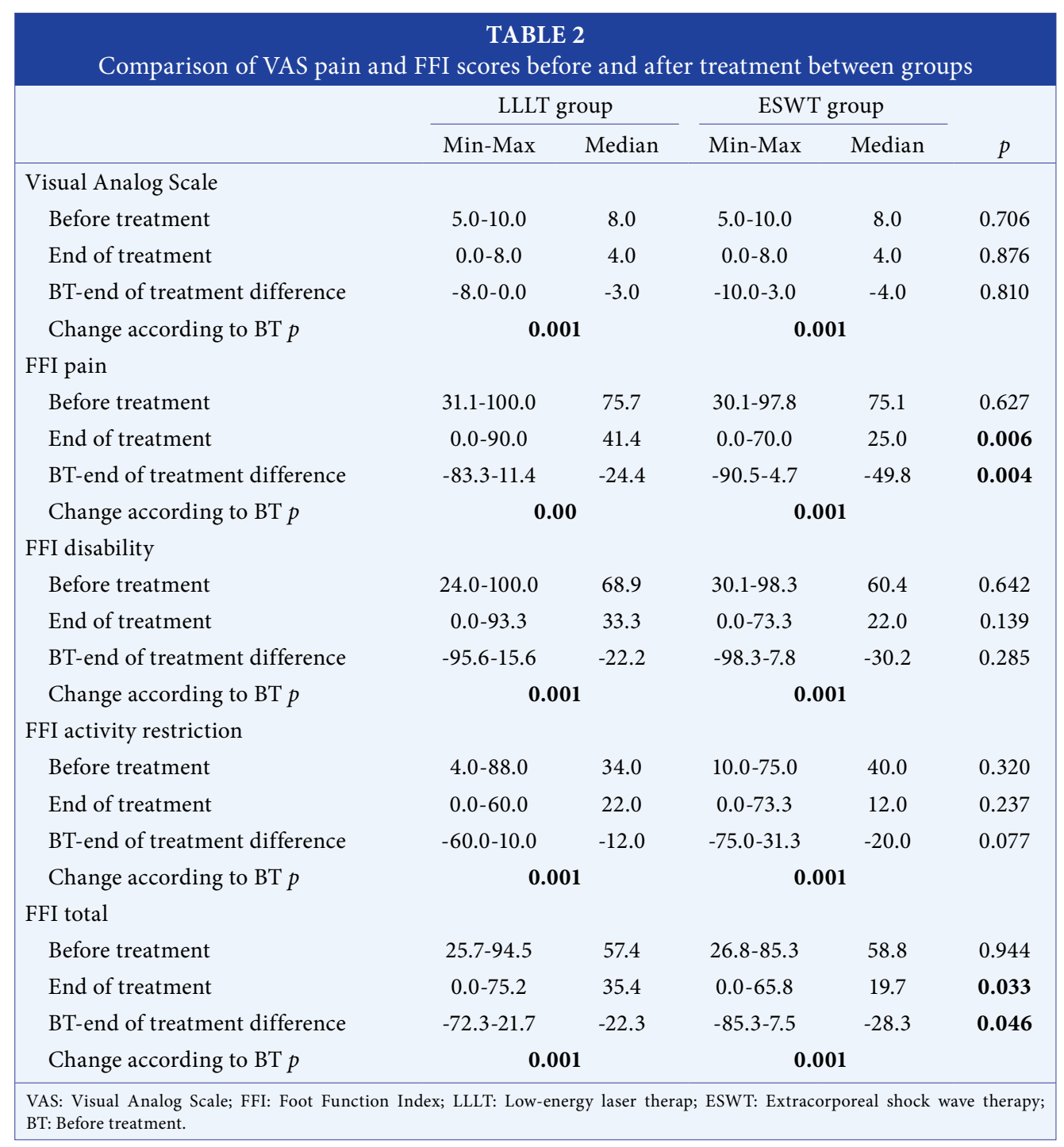

significant difference between the groups in terms of VAS, FFI disability, and FFI activity limitation scores after the treatment $(\mathrm{p}>0.05)$. In the ESWT group, the median FFI pain and total scores after treatment were significantly lower than in the LLLT group ( $p=0.006$ and $p=0.033$, respectively). The difference in the median FFI pain and total scores between before and after treatment was significantly higher in ESWT group than the LLLT group $(\mathrm{p}=0.004$ and $\mathrm{p}=0.046$, respectively) (Table 2).

\section{DISCUSSION}

In this study, we compared the efficacy of ESWT and LLLT in CS and found that both treatment modalities were effective and not superior to each other in terms of the FFI disability and activity limitation scores. However, the improvement in the median FFI pain and total scores after treatment were higher in the ESWT group than in the LLLT group. Many different treatment strategies have been proposed in CS and there is still a limited number of evidences regarding the efficacy of these treatment protocols. Therefore, we believe that our study provides additional contribution to the literature on the treatment of CS.

The ESWT has become an increasingly common treatment in the fields of surgery, orthopedics, rheumatology, and physical therapy in recent years. Physically, the shock wave is a low-frequency, instantaneous pressure disturbance mechanical (acoustic) wave that spreads rapidly through space. To date, numerous clinical studies have been conducted on the efficacy of ESWT in $\mathrm{CS}^{[1,10,13]}$ and have become the recommended method in recent years in patients with symptomatic CS, particularly in patients resistant 
to conservative treatment. Similarly, our study showed statistically significant effects of ESWT on both pain and FFI scores.

Although the biological mechanisms of the analgesic effect of ESWT are still controversial, beneficial effects have been proposed to be associated with accelerating micro-destruction and neovascularization. ${ }^{[21]}$ Tissue healing is stimulated by the release of local growth factors. ${ }^{[22]}$ The success rate of this non-invasive treatment with a very low complication rate has been reported to range between 75 and $94 \%$ in plantar fasciitis, and Lee et al. ${ }^{[23]}$ reported a success rate of $82 \%$ in patients with CS at 12 months in their study. Krukowska et al. ${ }^{[24]}$ also compared the efficacy of ultrasound and ESWT in CS and suggested that the analgesic efficacy of both treatments was similar and that ESWT could be superior with the advantage of requiring fewer sessions. A meta-analysis showed that ESWT reduced pain by $60 \%$ in plantar fasciitis, and that five of six studies reported superiority of ESWT over placebo. ${ }^{[25]}$ In another meta-analysis, ESWT was shown to be a preferable treatment modality, providing positive shortterm effects in plantar fasciitis. ${ }^{[9]}$

Low-level laser therapy is a non-invasive treatment modality which uses laser light at low levels and has gained popularity in recent years in the treatment of musculoskeletal diseases. The efficacy of LLLT in plantar fasciitis was first demonstrated in 1998 by Basford et al. ${ }^{[26]}$ and, in recent years, it has been used to reduce pain caused by plantar fasciitis. ${ }^{[27]}$ Experimental studies have shown that LLLT has anti-inflammatory and analgesic effects, ${ }^{[28]}$ and that it leads to tissue healing by increasing local microcirculation. ${ }^{[29]}$ It has been reported that LLLT has an analgesic effect and this effect may be due to the selective inhibition of nociceptive signals in peripheral nerves and, also, the power of initiating biophysical events in nerve tissue. ${ }^{[30]}$ Factors such as wavelength, power, frequency, application site, and duration are important in the evaluation of response to treatment.

In a prospective, clinical study, pain was significantly reduced in plantar fasciitis with LLLT. ${ }^{[27]}$ Cinar et al. ${ }^{[31]}$ also reported that LLLT significantly improved the American Orthopedic Foot and Ankle Society Score function subscale in three weeks and could increase walking distance effectively. In addition, ultrasound imaging results showed that LLLT significantly reduced plantar fascia thickness, compared to placebo. ${ }^{[32]}$ Contradictory to these results, some studies have suggested that LLLT in plantar fasciitis is not effective in reducing pain, ${ }^{[12,26]}$ although this controversy may be due to differences in the treatment protocols used. Basford et al. ${ }^{[26]}$ reported that the treatment protocols were $1 \mathrm{~J}$ for calcaneal origin and $2 \mathrm{~J}$ for the facial arch, whereas Ulusoy et al. ${ }^{[13]}$ applied $8 \mathrm{~J}$ of LLLT to the medial calcaneal area and myofascial junction in plantar fasciitis patients and concluded that this treatment was effective on both pain and functional outcomes. We used the latter treatment protocol in our study and, at the end of treatment, we found a significant decrease in the VAS-pain and FFI scores in the LLLT group. However, unlike the results of Ulusoy et al., ${ }^{[13]}$ we found a significantly higher improvement in the median FFI total scores in the ESWT group than in the LLLT group. Differences in the patient selection may have caused this discrepancy. In a meta-analysis including only prospective, randomized-controlled, clinical trials, LLLT was shown to be effective in reducing short-term heel pain, ${ }^{[8]}$ consistent with our findings. Therapeutic administration of LLLT involves multiple variables, such as dose, site of application and frequency, and the most appropriate treatment protocol has not been established, yet. ${ }^{[33]}$ Therefore, the importance of further prospective studies focusing on the most appropriate treatment parameters cannot be denied. Moreover, to the best of our knowledge, there are no studies evaluating the efficacy of LLLT specifically in CS, highlighting the importance of the present study in which we compared the efficacy of ESWT and LLLT, a treatment modality with proven efficacy in CS.

The main limitations of this study are the lack of long-term follow-up results and the absence of a control group. Nonetheless, we believe that these drawbacks do not outweigh the contributory value of this study.

In conclusion, both treatment modalities are effective and not superior to each other in terms of disability and activity limitation reduction, although a greater improvement in the FFI pain and total scores can be achieved with the ESWT. Based on these findings, we recommend both non-invasive treatment methods to be used in the treatment of calcaneal spurs in the clinical practice. However, further large-scale, long-term, prospective, randomized, placebo-controlled clinical trials are required to investigate the long-term efficacy and optimal treatment protocol of LLLT and ESWT in CS. 


\section{Declaration of conflicting interests}

The authors declared no conflicts of interest with respect to the authorship and/or publication of this article.

\section{Funding}

The authors received no financial support for the research and/or authorship of this article.

\section{REFERENCES}

1. Yalcin E, Keskin Akca A, Selcuk B, Kurtaran A, Akyuz M. Effects of extracorporal shock wave therapy on symptomatic heel spurs: a correlation between clinical outcome and radiologic changes. Rheumatol Int 2012;32:343-7.

2. McPoil TG, Martin RL, Cornwall MW, Wukich DK, Irrgang JJ, Godges JJ. Heel pain--plantar fasciitis: clinical practice guildelines linked to the international classification of function, disability, and health from the orthopaedic section of the American Physical Therapy Association. J Orthop Sports Phys Ther 2008;38:A1-A18.

3. Aldridge T. Diagnosing heel pain in adults. Am Fam Physician 2004;70:332-8.

4. McMillan AM, Landorf KB, Barrett JT, Menz HB, Bird AR. Diagnostic imaging for chronic plantar heel pain: a systematic review and meta-analysis. J Foot Ankle Res 2009;2:32.

5. Toomey EP. Plantar heel pain. Foot Ankle Clin 2009;14:229-45.

6. Yinilmez Sanmak ÖD, Geler Külcü D, Mesci N, Altunok EÇ. Comparison of effects of low-level laser therapy and extracorporeal shock wave therapy in plantar fasciitis treatment: A randomized, prospective, single-blind clinical study. Turk J Phys Med Rehabil 2018;65:184-90.

7. Jerosch J, Schunck J, Liebsch D, Filler T. Indication, surgical technique and results of endoscopic fascial release in plantar fasciitis (E FRPF). Knee Surg Sports Traumatol Arthrosc 2004;12:471-7.

8. Li X, Zhang L, Gu S, Sun J, Qin Z, Yue J, et al. Comparative effectiveness of extracorporeal shock wave, ultrasound, low-level laser therapy, noninvasive interactive neurostimulation, and pulsed radiofrequency treatment for treating plantar fasciitis: A systematic review and network meta-analysis. Medicine (Baltimore) 2018;97:e12819.

9. Yin MC, Ye J, Yao M, Cui XJ, Xia Y, Shen QX, et al. Is extracorporeal shock wave therapy clinical efficacy for relief of chronic, recalcitrant plantar fasciitis? A systematic review and meta-analysis of randomized placebo or active-treatment controlled trials. Arch Phys Med Rehabil 2014;95:1585-93.

10. Hayta E, Salk I, Gumus C, Tuncay MS, Cetin A. Extracorporeal shock-wave therapy effectively reduces calcaneal spur length and spur-related pain in overweight and obese patients. J Back Musculoskelet Rehabil 2016.

11. Ermutlu C, Aksakal M, Gümüştaş A, Özkaya G, Kovalak E, Özkan Y. Thickness of plantar fascia is not predictive of functional outcome in plantar fasciitis treatment. Acta Orthop Traumatol Turc 2018;52:442-6.
12. Macias DM, Coughlin MJ, Zang K, Stevens FR, Jastifer JR, Doty JF. Low-Level Laser Therapy at $635 \mathrm{~nm}$ for Treatment of Chronic Plantar Fasciitis: A Placebo-Controlled, Randomized Study. J Foot Ankle Surg 2015;54:768-72.

13. Ulusoy A, Cerrahoglu L, Orguc S. Magnetic resonance imaging and clinical outcomes of laser therapy, ultrasound therapy, and extracorporeal shock wave therapy for treatment of plantar fasciitis: a randomized controlled trial. J Foot Ankle Surg 2017;56:762-7.

14. Yinilmez Sanmak ÖD, Geler Külcü D, Mesci N, Altunok EÇ. Comparison of effects of low-level laser therapy and extracorporeal shock wave therapy in plantar fasciitis treatment: A randomized, prospective, single-blind clinical study. Turk J Phys Med Rehabil 2018;65:184-90.

15. Kiritsi O, Tsitas K, Malliaropoulos N, Mikroulis G. Ultrasonographic evaluation of plantar fasciitis after low-level laser therapy: results of a double-blind, randomized, placebo-controlled trial. Lasers Med Sci 2010;25:275-81.

16. Rola P, Doroszko A, Derkacz A. The use of low-level energy laser radiation in basic and clinical research. Adv Clin Exp Med 2014;23:835-42.

17. Bjordal JM, Couppé C, Chow RT, Tunér J, Ljunggren EA. A systematic review of low level laser therapy with locationspecific doses for pain from chronic joint disorders. Aust J Physiother 2003;49:107-16.

18. World Association for Laser Therapy (WALT). Dosage Recommendations and Scientific Guidelines. Available at: http://www.walt.nu/dosagerecommendationsand-scientificguidelines. [Accessed: March 15, 2010]

19. Marvin JA. Pain assessment versus measurement. J Burn Care Rehabil 1995;16:348-57.

20. Anaforoğlu Külünkoğlu B, Fırat N, Yıldız NT, Alkan A. Reliability and validity of the Turkish version of the Foot Function Index in patients with foot disorders. Turk J Med Sci 2018;48:476-83.

21. Chow IH, Cheing GL. Comparison of different energy densities of extracorporeal shock wave therapy (ESWT) for the management of chronic heel pain. Clin Rehabil 2007;21:131-41.

22. Thiel M. Application of shock waves in medicine. Clin Orthop Relat Res 2001;(387):18-21.

23. Lee GP, Ogden JA, Cross GL. Effect of extracorporeal shock waves on calcaneal bone spurs. Foot Ankle Int 2003;24:927-30.

24. Krukowska J, Wrona J, Sienkiewicz M, Czernicki J. A comparative analysis of analgesic efficacy of ultrasound and shock wave therapy in the treatment of patients with inflammation of the attachment of the plantar fascia in the course of calcaneal spurs. Arch Orthop Trauma Surg 2016;136:1289-96.

25. Aqil A, Siddiqui MR, Solan M, Redfern DJ, Gulati V, Cobb JP. Extracorporeal shock wave therapy is effective in treating chronic plantar fasciitis: a meta-analysis of RCTs. Clin Orthop Relat Res 2013;471:3645-52.

26. Basford JR, Malanga GA, Krause DA, Harmsen WS. A randomized controlled evaluation of low-intensity laser therapy: plantar fasciitis. Arch Phys Med Rehabil 1998;79:249-54. 
27. Jastifer JR, Catena F, Doty JF, Stevens F, Coughlin MJ. Low-level laser therapy for the treatment of chronic plantar fasciitis: a prospective study. Foot Ankle Int 2014;35:566-71.

28. Boschi ES, Leite CE, Saciura VC, Caberlon E, Lunardelli A, Bitencourt S, et al. Anti-Inflammatory effects of low-level laser therapy $(660 \mathrm{~nm})$ in the early phase in carrageenaninduced pleurisy in rat. Lasers Surg Med 2008;40:500-8.

29. Bingöl U, Altan L, Yurtkuran M. Low-power laser treatment for shoulder pain. Photomed Laser Surg 2005;23:459-64.

30. Jarvis D, MacIver BM, Tanelian DL. Electrophysiologic recording and thermodynamic modeling demonstrate that helium-neon laser irradiation does not affect peripheral
Adelta- or C-fiber nociceptors. Pain 1990;43:235-42.

31. Cinar E, Saxena S, Uygur F. Low-level laser therapy in the management of plantar fasciitis: a randomized controlled trial. Lasers Med Sci 2018;33:949-58.

32. Salvioli S, Guidi M, Marcotulli G. The effectiveness of conservative, non-pharmacological treatment, of plantar heel pain: A systematic review with meta-analysis. Foot (Edinb) 2017;33:57-67.

33. Wang W, Jiang W, Tang C, Zhang X, Xiang J. Clinical efficacy of low-level laser therapy in plantar fasciitis: A systematic review and meta-analysis. Medicine (Baltimore) 2019;98:e14088. 